\title{
Comparison of methods to determine load sharing of insulating glass units for environmental loads
}

\author{
Stephen M. Morse - H. Scott Norville
}

Received: 31 December 2015 / Accepted: 28 April 2016 / Published online: 10 May 2016

(C) Springer International Publishing Switzerland 2016

\begin{abstract}
In the past few decades, designers increasingly work on glazing projects across the world requiring the use of international glazing standards that often differ from the designer's national code. Commonly specified international glazing standards include prEN16612, AS1288, and ASTM E1300. Each of these standards has a different approach to determine window glass strength. This paper explores one aspect of glazing design for insulating glass units, the method for estimating the load sharing between the lites comprising insulating glass units. Each glazing standard uses a different method for estimating load sharing and how the effects of environmental loads are incorporated into the estimation. Environmental loads include but are not limited to changes in atmospheric pressure, resulting from elevation, climatic, and temperature variations. Additionally, several iterative methods appear in technical literature that attempt to account for most known factors affecting insulating glass load sharing. Each of these methods addresses environmental loads differently with differing degrees of accuracy. This paper presents comparisons between the three glazing standards above and an iterative method for load sharing with environmental loads in double and triple glazed insulating glass units. The major factors varied in the investigation are insulating glass unit constructions, dimensions, glass lite thicknesses, air space thickness, temperature and pressure and atmospheric
\end{abstract}

S. M. Morse $(\varangle) \cdot$ H. S. Norville

Texas Tech University, Lubbock, USA

e-mail: stephen.m.morse@ttu.edu pressure changes due to elevation change. Pressure versus load sharing percentage curves are presented for select double and triple insulating glass unit constructions. Figures in this paper will show the load sharing trends of different similarly loaded insulating glass units due to varying the pressure differential between the air space(s) and atmospheric pressures. The results of this study will indicate differences between the load sharing methods and provide example scenarios where the methods produce similar and different estimates of insulating glass load sharing.

Keywords Insulating glass units - Load sharing · Environmental loads

\section{Introduction}

Insulating glass units (IGU), consisting of two glass lites separated by a sealed air space, commonly referred to as a double glazed IGU (DIGU), have been widely used in commercial and residential construction since the 1960's to provide more thermal, sound and solar insulation than possible with single glazed fenestration. In response to energy polices requiring buildings to use less energy, the use of triple glazed IGUs (TIGU), consisting of three glass lites separated by two sealed air spaces, has increased due to their improved insulating performance over DIGUs. Extensive research on load sharing between the lites comprising a DIGUs appears in technical literature, whereas, the load shar- 
ing between the lites comprising a TIGUs has not been extensively studied. Many designers and specifiers often design TIGUs as a DIGU by ignoring the contribution of the middle lite to load sharing. Additionally, commonly used window glass strength standards, e.g. ASTM E 1300 (ASTM 2012), AS1288 (SAS 2006), have extended the so-called "thickness cubed" method to TIGUs to apportion the load carried by each lite comprising the IGU. This simplistic approach is tenuously valid for DIGUs when only considering the load sharing due to an applied external pressure (Morse and Norville 2015). However, when net pressure changes between the sealed air space(s) and atmospheric pressure due to temperature, elevation, and barometric pressure variations are accounted for with DIGUs and especially TIGUs, this simplistic approach becomes invalid. A method must ensure the ideal gas law is in equilibrium for each sealed air space(s) and the displaced volume of the loaded lites in order for the method to accurately predict the load sharing between the lites. This paper presents a comparison of the load sharing methods from three commonly used window glass strength standards, ASTM E 1300-12 ${ }^{\text {a } 1}$, AS 1288-06, and prEN 16612 (EN 2013) and an advanced iterative method advanced by Norville and Morse (2011). To limit additional variations between the methods, only annealed monolithic lites will addressed in the comparisons herein. These comparisons will be of particular interest to designers who specify IGUs sealed from the manufacturer, usually due to the air space(s) filled with an alternate gas, such as argon, and IGUs with surface coatings that significantly increase the temperature of the air space(s) such as Low-E coatings and ceramic frits.

\section{Background}

The term load sharing ratio (LSR) will be used herein to provide an unambiguous name to represent the various terms and symbols used for load sharing in the four methods. The LSR is defined as the ratio of the load carried by an individual lite of an IGU and the applied external load. The LSR incorporates the pressure differential between the atmospheric pressure and the pressure in the sealed air space(s) and the coupled change in air space(s) volume due to the lateral displacement of the lites comprising the IGU. In general, the summation of the LSRs for all lites comprising an IGU should equate to 1.0. However, two methods use a scaling factor to increase the load attributed to each lite; these cases will be elucidated in the following sections. A LSR with a magnitude greater than 1.0 indicates a pressure differential exists between the atmospheric pressure and the pressure in the sealed air space(s).

\subsection{ASTM E1300-12 $2^{\mathrm{a} \varepsilon 1}$}

ASTM E $1300-12^{\mathrm{a} \varepsilon 1}$ is designed to determine the load resistance of a given glazing construction. The standard provides calculated load share factors (LSF) shown in Tables 5 and 6 of the standard's normative provisions and are based on the minimum lite thicknesses from ASTM C 1036-11 (ASTM 2011). The inverse of the thickness cubed method is used to calculate the load share factors in Table 5. A modified version of the thickness cubed method to address effects of laminated glass in IGUs with externally applied long duration loads, such as a snow load, is used for Table 6. For a DIGU comprised of monolithic annealed lites of equal thickness, the load share factor is 2.0 for both lites. To account for the effects of a net pressure differential between the atmospheric pressure and IGU air space(s), a factor has been incorporated into the glass type factors provided in ASTM E 1300-12 ${ }^{\text {a } \varepsilon 1}$ Tables 2 and 7 for DIGUs and TIGUs, respectively. The rational for the factor is presented in Appendix X2 of ASTM E 1300-12 $2^{\mathrm{a} 11}$. In short, for DIGUs comprised of monolithic annealed lites, the factor is 0.9 , and for TIGUs comprised of monolithic annealed lites, the factor is 0.81 . Equations (1) and (2) present the relationship to translate the factors presented in ASTM E 1300-12 ${ }^{\mathrm{a} \varepsilon 1}$ to a LSR for DIGUs and TIGUs, respectively. Where i denotes the ith lite of the IGU.

$$
\begin{aligned}
L S R_{i} & =\frac{1}{0.9 \cdot L S F_{i}} \\
L S R_{i} & =\frac{1}{0.81 \cdot L S F_{i}}
\end{aligned}
$$

\subsection{AS 1288-06}

AS 1288-06 also uses the thickness cubed method to determine the load apportioned to each lite comprising an IGU. A factor of 1.25 has also been added to the thickness cubed method used in AS 1288-06, presumably to account for atmospheric effects, although 
it is not specifically defined as such within the standard. Equation (3) presents the relationship to translate the factored thickness cubed method presented in AS 1288-06 to a LSR for IGUs. Where i denotes the ith lite of the IGU and $n$ denotes the total number of lites comprising the lite.

$L S R_{i}=\frac{1.25 \cdot t_{i}^{3}}{\sum_{i}^{n} t_{i}^{3}}$

Additionally, AS 1288-06 specifies different minimum thicknesses for each nominal thickness designation than specified in ASTM E 1300-12 ${ }^{\text {a1 }}$. To limit additional variations to the LSRs due to the differential in minimum thicknesses, the minimum thicknesses specified in ASTM E 1300-12 ${ }^{\text {a } 1}$ will be used herein. Interestingly, the inverse of the 1.25 factor is 0.80 , which is very close to the TIGU glass type factor specified in ASTM E $1300-12^{\mathrm{a} \varepsilon 1}$. However, the authors opine the factors were created independently and likely based on different rationales.

\section{3 prEN 16612}

The method in prEN 16612 to determine the apportioned externally applied load for each lite comprising an IGU is based on work advanced by Siebert and Maniatis (2012). The prEN 16612 method ensures the sealed air space(s) of an IGU is in equilibrium with the ideal gas law. The prEN 16612 method uses a simplified linear method to determine the volume change of a uniformly loaded lite. Furthermore, this procedure allows for lite shapes other than rectangular by providing alternate uniform load and volume change relationships. The procedure is essentially the same for both DIGUs and TIGUs and can be extended to IGUs with $n$ number of lites and $n-1$ air spaces. Again, to minimize additional variations the minimum thicknesses specified in ASTM E $1300-12^{\mathrm{a} \varepsilon 1}$ will be used. The prEN 16612 contains different formulations for determining the load carried by each lite comprising IGUs for DIGUs and TIGUs. Furthermore, the contribution of load from external loads and climatic loads have been separated to allow for the use of separate factors, e.g. load duration, etc. Equations (4) and (5) present the formulations for determining the combined external, e.g. wind, snow, and the summation of the climatic loads resulting from elevation, temperature and local atmospheric pressure changes for DIGUs.

$$
\begin{aligned}
& P_{1}=P_{e x, 1} \cdot\left(\delta_{1}+\phi \cdot \delta_{2}\right)-\phi \cdot P_{0} \\
& P_{2}=P_{e x, 1} \cdot\left(\delta_{2} \cdot(1-\phi)\right)+\phi \cdot P_{0}
\end{aligned}
$$

where $\mathrm{P}_{1}$ and $\mathrm{P}_{2}$ denote the load carried by lites 1 and 2 , respectively; $\mathrm{P}_{e x, 1}$ denotes the external uniform pressure applied to lite $1 ; \delta_{1}$ and $\delta_{2}$ denote the stiffness partitions for lites 1 and 2, respectively; $\phi$ denotes the insulating unit factor and $\mathrm{P}_{0}$ denotes the summation of the climatic loads shown in Eq. (6).

$$
\begin{aligned}
P_{0}= & 0.012 \frac{k P a}{m} \cdot(\Delta H)+0.34 \frac{k P a}{{ }^{\circ} K} \cdot(\Delta T) \\
& -\left(P_{a}-P_{p}\right)
\end{aligned}
$$

where $\Delta \mathrm{H}$ denotes the change in elevation between IGU assembly and installation, $\Delta \mathrm{T}$ denotes the change in temperature between IGU assembly and installation, $\mathrm{P}_{a}$ denotes the pressure of the airspace when the IGU was assembled, and $\mathrm{P}_{p}$ denotes the change in pressure at the installed location due to meteorological events. For brevity equations for $\delta_{1}, \delta_{2}$, and $\phi$ are not repeated here and can be found in prEN16612.

Equations (7), (8) and (9) present the formulations for determining the combined external, e.g. wind, snow, and the summation of the climatic loads resulting from elevation, temperature and local atmospheric pressure changes for TIGUs.

$P_{1}=P_{e x, 1}-\Delta P_{w, 1}+\Delta P_{c, 1}$

$P_{2}=\Delta P_{w, 1}-\Delta P_{w, 2}+\Delta P_{c, 1}-\Delta P_{c, 2}$

$P_{3}=\Delta P_{w, 2}-\Delta P_{c, 2}$

where $\mathrm{P}_{1}, \mathrm{P}_{2}$ and $\mathrm{P}_{3}$ denote the load carried by lites 1,2 and 3 , respectively; $\mathrm{P}_{e x, 1}$ denotes the uniform external pressure applied to lite $1 ; \Delta \mathrm{P}_{w, 1}$ and $\Delta \mathrm{P}_{w, 2}$ denote the net pressure difference across air space 1 and 2 , respectively, due to the applied external uniform load presented in Eqs. (10) and (11); and $\Delta \mathrm{P}_{c, 1}$ and $\Delta \mathrm{P}_{c, 2}$ denote the net pressure difference across air space 1 and 2 , respectively, due to the summation of the climatic loads resulting from elevation, temperature and local atmospheric pressure changes presented in Eqs. (12) and (13).

$$
\begin{aligned}
& \Delta P_{w, 1}=P_{e x, 1} \cdot\left(\frac{\phi_{1} \cdot \alpha_{1}}{\beta}\right) \\
& \Delta P_{w, 2}=P_{e x, 1} \cdot\left(\frac{\phi_{1} \cdot \alpha_{1} \cdot \phi_{2} \cdot \alpha_{2}}{\beta}\right)
\end{aligned}
$$




$$
\begin{aligned}
& \Delta P_{c, 1}=P_{0} \cdot \phi_{1} \cdot\left(\frac{1+\phi_{2} \cdot \alpha_{1}^{+}}{\beta}\right) \\
& \Delta P_{c, 2}=P_{0} \cdot \phi_{2} \cdot\left(\frac{1+\phi_{1} \cdot \alpha_{2}}{\beta}\right)
\end{aligned}
$$

where $\phi_{1}$ and $\phi_{2}$ denote the insulating unit factor for air space 1 and 2 , respectively, and $\alpha_{1}$ and $\alpha_{2}$ denote the relative volume change for air space 1 and 2 , respectively. For brevity equations for $\phi_{1}, \phi_{2}, \alpha_{1}, \alpha_{2}$, and $\beta$ are not repeated here and can be found in prEN16612.

As this method directly estimates the apportioned externally applied load for each lite comprising an IGU the LSR will be taken as the ratio of the load carried by an individual lite of an IGU and the applied external load, as presented in Eq. (14).

$$
L S R_{i}=\frac{\mathrm{P}_{i}}{P_{\text {ex }, 1}}
$$

\subsection{Iterative method}

This method, first advanced by Vallabhan and Chou (1986) for DIGUs and extended by Norville and Morse (2011) addresses IGUs with $\mathrm{n}$ number of lites and $\mathrm{n}-1$ air spaces, by iteratively converging on an equilibrium between the air space(s) governed by the ideal gas law and non-dimensionalized load/volume interaction curves derived from a non-linear model for uniformly laterally loaded rectangular plates for large deflections. The use of the ideal glass law ensures the airspace pressure(s) are in equilibrium with the applied external load, pressure differential due to the climatic variations and the stiffness of the plates. For TIGUs, the two airspaces typically have different pressures, even for symmetric configurations, as illustrated in Sect. 3 . To minimize additional variations due to different lite thicknesses specified in other methods, the minimum thicknesses specified in ASTM E 1300-12 a ${ }^{\text {a1 }}$ will be used. As this method also directly estimates the apportioned externally applied load for each lite comprising an IGU, the LSR will be taken as the ratio of the load carried by an individual lite of an IGU and the applied external load as presented in Eq. (14).

\subsection{Pressure differential between atmospheric and air space pressures}

For the purposes of this study, it is assumed the IGU air space(s) are sealed so no amount of gas enters or leaves the air space(s) and the pressure of the air space(s) equals the atmospheric pressure when the air space(s) are sealed. Several environmental conditions including changes in elevation, temperature of the environment and/or the air space(s), and local barometric pressure can cause a pressure differential between the atmospheric and air space pressures. Regardless of the environmental condition(s), only the resulting pressure differential actually creates a load on the lites comprising the IGU. To simplify the presentation of the comparison between the methods and to eliminate variations caused by differences in the calculation of the $\mathrm{P}_{0}$ resulting from environmental conditions, the comparisons will be explored with $\mathrm{P}_{0}$ ranging from -15 to 15 $\mathrm{kPa}$ with $5 \mathrm{kPa}$ increments.

\section{Method comparison}

\subsection{Loading cases}

Eight loading cases result by varying the direction the atmospheric pressure differential, the applied external load orientation and the lite the applied external load acts. The $\mathrm{P}_{0}$ can be larger or smaller than the initial air space pressure(s). The direction of the applied load can be directed either into the IGU or away from it and the applied load can act on either external lite, as illustrated in Fig. 1. Cases 1, 3, 5, 7 have a lower atmospheric pressure than the air space pressure(s) resulting in the lite spreading apart or "pillowing", represented as $\mathrm{P}_{0}$ with
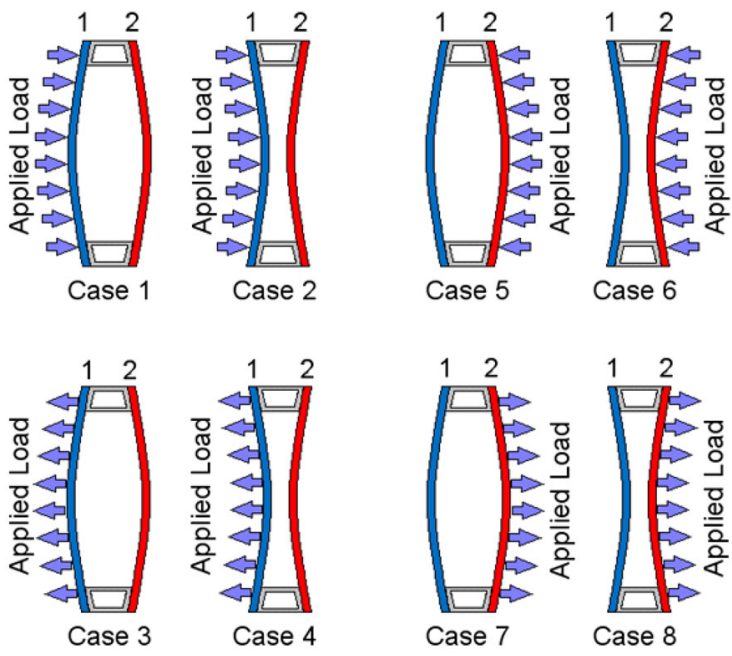

Fig. 1 Loading cases 
Table 1 IGU comparator constructions

\begin{tabular}{ll}
\hline IGU comparator & Construction $(\mathrm{mm})$ \\
\hline DIGU1 & $1500 \times 1000 \times(3.78|12| 3.78)$ \\
DIGU2 & $1500 \times 1000 \times(3.78|12| 5.56)$ \\
DIGU3 & $500 \times 500 \times(5.56|12| 5.56)$ \\
TIGU1 & $2500 \times 1000 \times(3.78|12| 3.78|12| 3.78)$ \\
TIGU2 & $2200 \times 600 \times(2.59|12| 3.78|12| 4.57)$ \\
TIGU3 & $2200 \times 600(2.59|12| 4.57|12| 3.78)$ \\
\hline
\end{tabular}

a negative magnitude. Cases 2, 4, 6, 8 have a larger atmospheric pressure than the air space pressure(s), resulting in the lites pressing together, represented as $\mathrm{P}_{0}$ with a positive magnitude. For constructions comprised with lites of equal thickness, the number of loading case reduces to four cases $(1,2,3,4)$, as applying the load to the other lite will not produce different results. TIGUs will be treated in a similar manner (not shown in Fig. 1). Although the iterative methods provides the flexibility for each airspace to have independent temperatures, the other methods do not; therefore, this type of loading case will not be addressed here.

\subsection{IGU comparator constructions}

The selected comparator constructions listed in Table 1 will illustrate the effect IGU construction, rectangular dimensions, the direction and magnitude of the applied external load, and whether the atmospheric pressure magnitude is larger or smaller than the air space pressure has on load sharing between the lites comprising an IGU. The IGU comparator constructions listed in Table 1 follow the format [long dimension X short dimension X (Lite 1 thickness|air space 1 thickness|Lite 2 thickness] and for TIGU comparators the addition of[|air space 2 thickness|Lite 3 thickness]. Despite only exploring a few of the enumerable number of IGU comparator constructions, several trends will become apparent.

\subsection{LSR comparison charts}

A series of charts were constructed to illustrate the variation of the LSRs for each loading case with external applied loads varying from 0.25 to $10 \mathrm{kPa}$ and $\mathrm{P}_{0}$ ranging between -15 and $15 \mathrm{kPa}$. While the $\mathrm{P}_{0}$ often has a magnitude smaller than $5 \mathrm{kPa}$ it can be higher than 15 $\mathrm{kPa}$ in some cases. The larger $\mathrm{P}_{0}$ magnitudes are principally included to provide insight into the change in LSR magnitudes relative to the $\mathrm{P}_{0}$ magnitude and the rate the effect of $\mathrm{P}_{0}$ on LSRs decreases as the magnitude of the external applied load increases. Due to the large number of LSR series resulting from the four methods for calculating LSRs and the eight loading cases considered, the LSRs corresponding to each IGU comparator have been divided into a set of four charts. The four charts include two charts for the LSRs calculated using the prEN 16612 method, one for loading cases 1 to 4 , and one for cases 5 to 8 . The remaining two charts for the LSRs were calculated using the iterative method, one for loading cases 1 to 4 and one for cases 5 to 8 . For symmetric IGU comparators (DIGU1, DIGU3, and TIGU1) only the charts for the loading cases 1 to 4 are presented as loading cases 5 to 8 do not have different values. Fig. 2 shows an example of a LSR comparison chart, for the DIGU1 comparator with LSRs calculated using the prEN 16612 method. A color-coded drawing of the IGU cross-section has been added to the charts located at the bottom right and bottom left corners to help distinguish the loading cases. The lites comprising the IGUs are numbered sequentially left to right. The lites in the drawing of the IGU cross-section are colorcoded with the corresponding colors. Lite 1 is always denoted with blue lines and the other exterior lite (Lite 2 for DIGU and Lite 3 for TIGU) is always denoted with red lines. For TIGUs, the middle lite (Lite 2) is denoted with green lines. The additional graphics for the TIGU comparators are not shown in Fig. 2 but are present in Fig. 9 through Fig. 18. Loading cases 1, 2, 5, 6 will always appear on the right side of the charts, as they have external applied loads directed into the IGU, represented as positive values. Loading cases 3, 4, 7, 8 will always appear on the left side of the charts, as they have external applied loads directed away from the IGU, represented as negative values. To distinguish the pairs of loading cases, the LSR series corresponding to loading cases for negative $\mathrm{P}_{0}(1,3,5,7)$ are represented with solid lines and LSR series corresponding to loading cases for positive $\mathrm{P}_{0}(1,3,5,7)$ are represented with dashed lines. The various $\mathrm{P}_{0}$ magnitudes are usually easily distinguishable with the largest $\mathrm{P}_{0}$ at the top and bottom extents of the charts and smaller magnitudes graduated towards the center of the charts. The $\mathrm{P}_{0}$ magnitudes are indicated with labels to help with orientation; however, the remaining charts do not have the 
Fig. 2 DIGU2-prEN 16612 load share ratios versus applied external uniform load magnitude for loading cases $1,2,3,4$
Fig. 3 DIGU1-prEN 16612 method load share ratios versus applied external uniform load magnitude for loading cases $1,2,3,4$
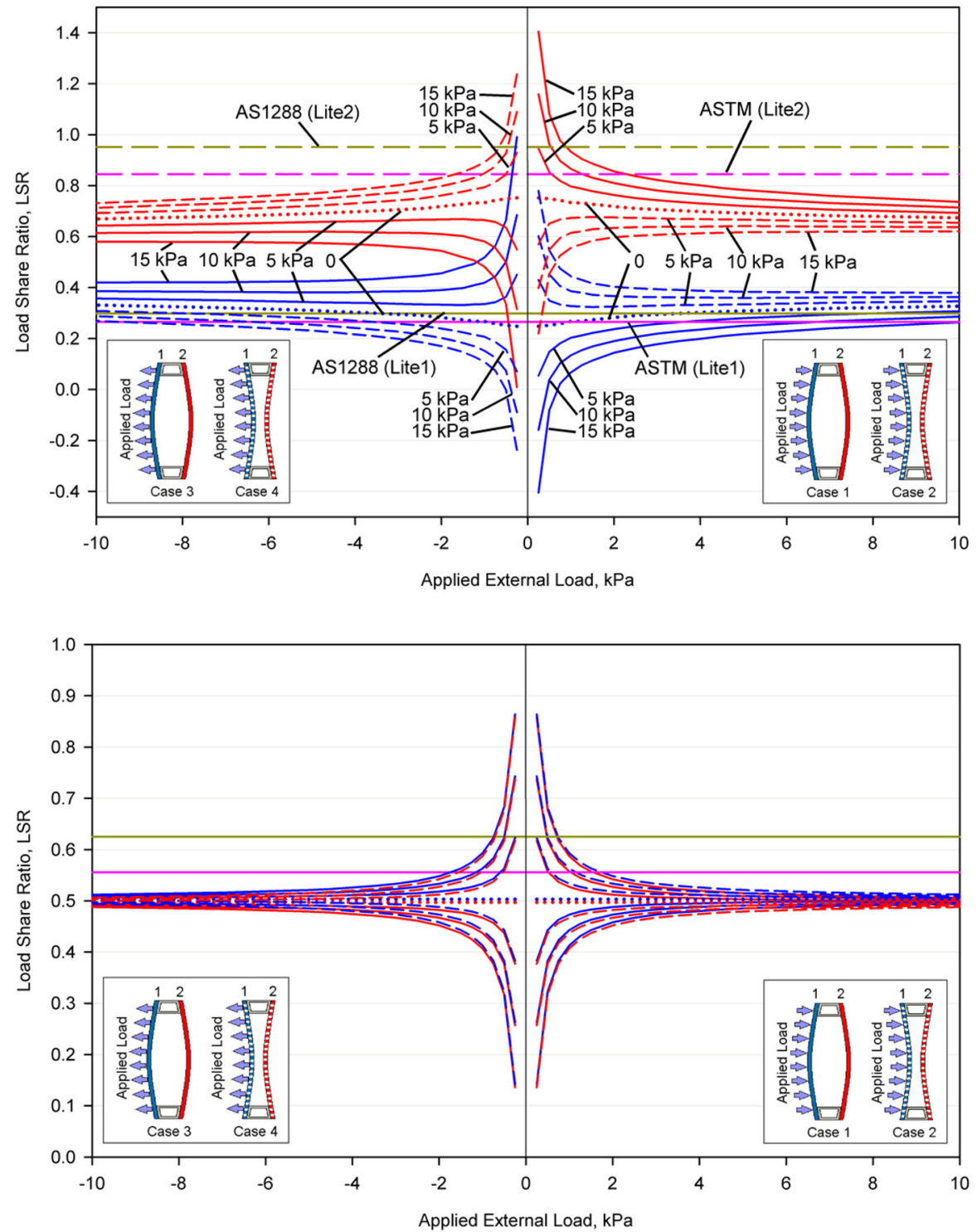

$\mathrm{P}_{0}$ magnitudes labeled so the chart can be clearly interpreted. As the LSR methods from ASTM E1300 and AS 1288 only vary with lite thickness, they are shown on each chart as horizontal lines (pink for ASTM E1300 and dark yellow for AS 1288), as shown in Fig. 2.

\section{Double insulating glass unit comparators}

\subsection{IGU comparator: DIGU1}

DIGU1 represents a small commercial or large residential size symmetric DIGU. Figures 3 and 4 show the LSRs calculated using the prEN16612 and the iterative methods, respectively. Loading cases 5, 6, 7, 8 are not presented due to symmetry. The LSRs values in Fig. 2 between loading cases 1 and 2 are nearly equal and opposite with respect to the lites, indicating the sign of the $\mathrm{P}_{0}$ only slightly affects the LSR magnitudes for this comparator. Loading cases 3 and 4 have exact mirror values with respect to the applied external load magnitudes compared to loading cases 2 and 1 , respectively, indicating the prEN16612 method only distinguishes between the load cases where the $\mathrm{P}_{0}$ sign and the applied external load direction are either in the same or opposite directions. 
Fig. 4 DIGU1-iterative method load share ratios versus applied external uniform load magnitude for loading cases $1,2,3,4$
Fig. 5 DIGU2-prEN 16612 method load share ratios versus applied external uniform load magnitude for loading cases $1,2,3,4$
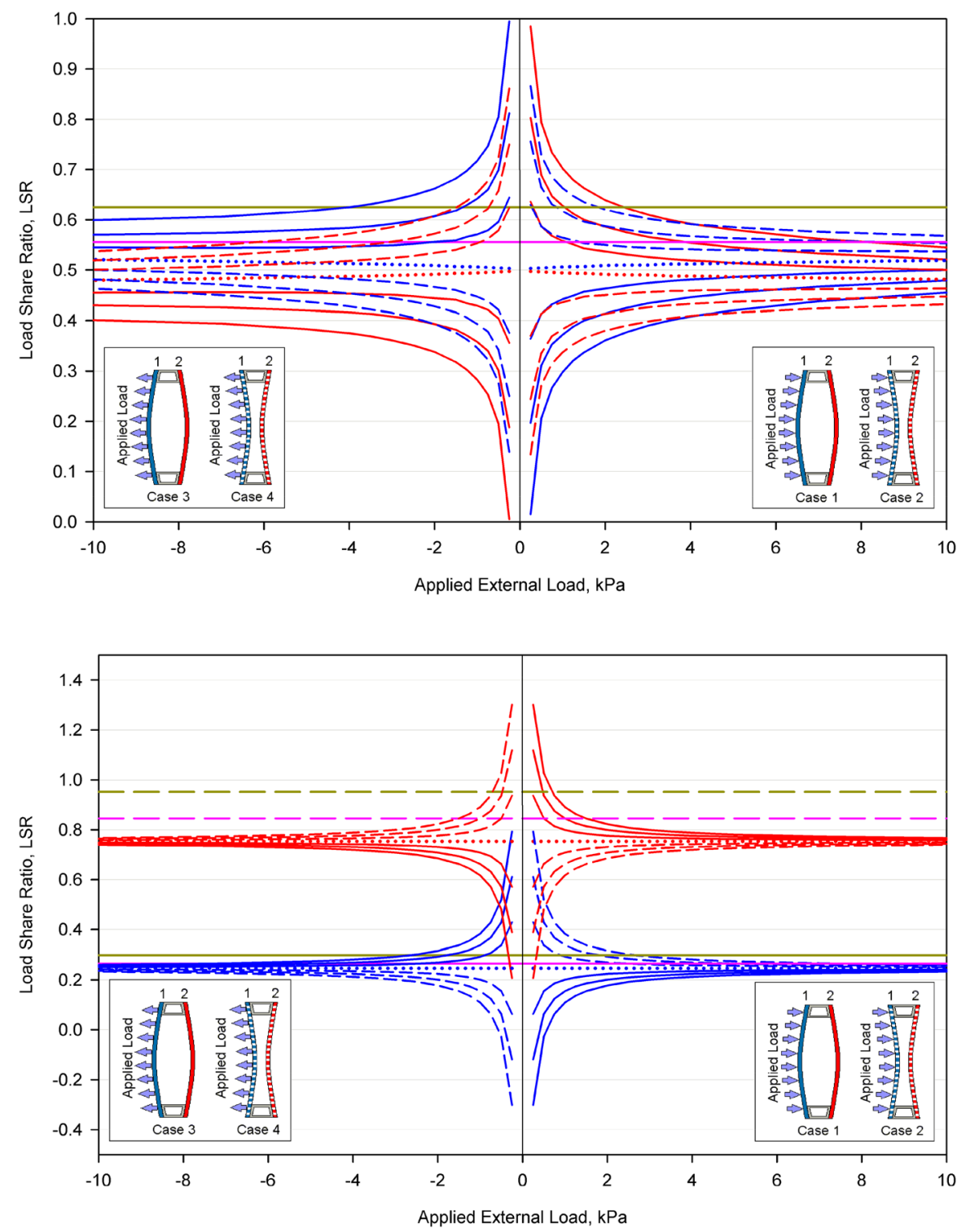

The LSRs values in Fig. 4 show similar trends to those presented in Fig. 3. However, greater disparity between loading cases 1 and 2, and loading cases 3 and 4 resulting in larger LSR magnitudes on Lite 1 exists; indicating the sign and magnitude of $\mathrm{P}_{0}$ affects the LSR values more in the iterative method than the prEN 16612 method. Furthermore, the LSR values between cases 3 and 4, and cases 2 and 1, respectively are not mirror LSR values as with the prEN 16612 LSRs. The LSR values have significantly differing magnitudes, especially at larger applied external load magnitudes, indicating each of the four combinations of the $\mathrm{P}_{0}$ sign and the applied external load direction result in different LSR values.

The LSR values shown in Figs. 3 and 4 for ASTM E1300 (shown in pink) are 0.556 for both lites and for AS 1288 (shown in dark yellow) are 0.625 for both lites. Ideally, these values should be larger than those determined with the other methods, and they are for applied external load magnitudes greater than $2.0 \mathrm{kPa}$ in Fig. 3. However, Fig. 3 suggests loading cases likely exist where ASTME1300 and AS 1288 methods do not sufficiently encompass all possible variations in LSR values. 
Fig. 6 DIGU2-prEN 16612 method load share ratios versus applied external uniform load magnitude for loading cases $5,6,7,8$
Fig. 7 DIGU2 - iterative method load share ratios versus applied external uniform load magnitude for loading cases $1,2,3,4$
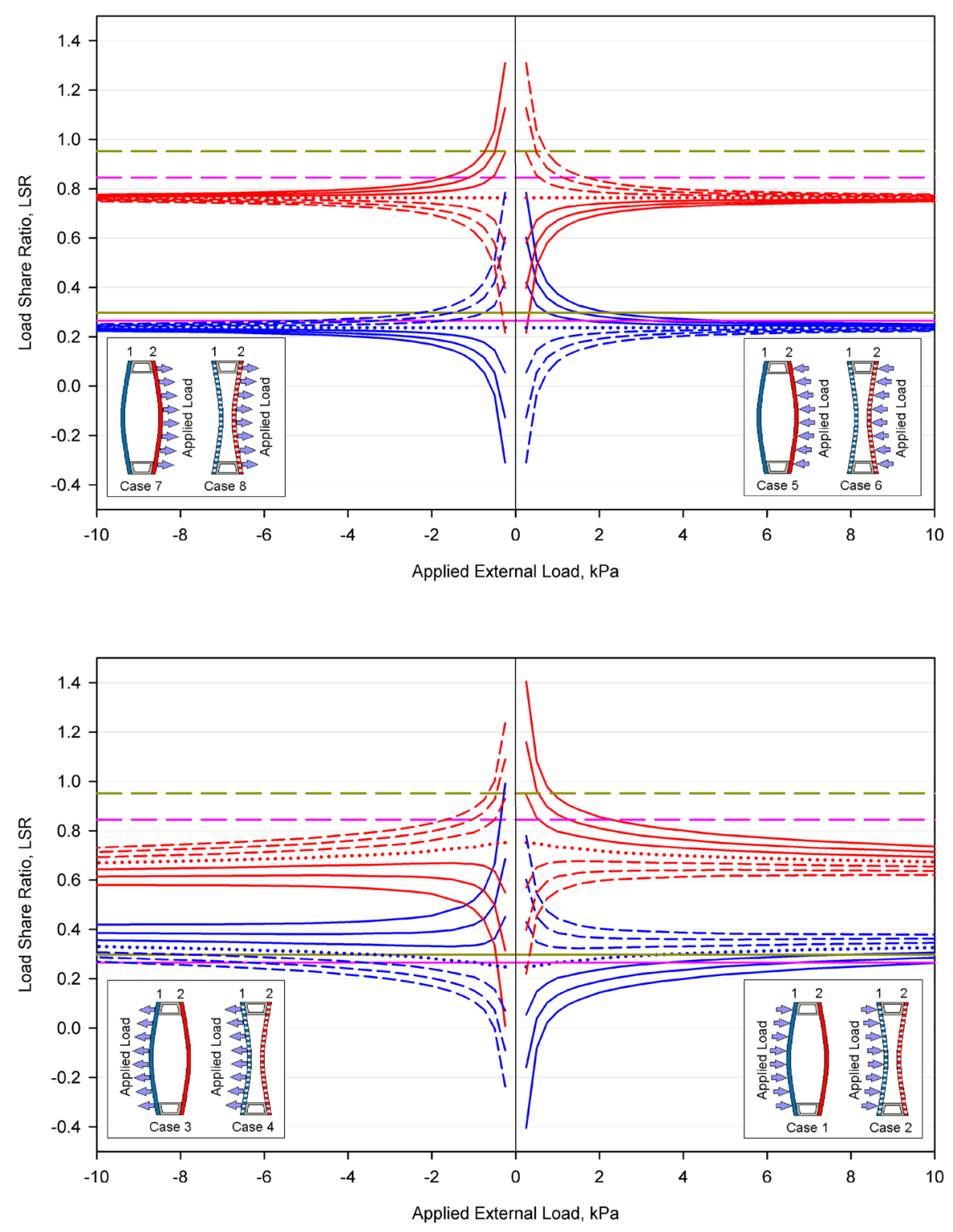

\subsection{IGU comparator: DIGU2}

DIGU2 represents a small commercial or large residential size asymmetric DIGU. Figures 5 and 6 present the eight loading cases for the prEN16612 LSRs and Figs. 7 and 8 present the eight loading cases for the iterative method LSRs. As expected, the LSRs for the thicker lite (Lite 2) are larger than Lite 1 indicating Lite 2 carries a larger portion of the applied external load than Lite 1 . Consistent with the LSRs shown in Figs. 2, 5 and 6 have exact mirror LSR values with respect to the applied external load mag- nitudes. The LSR series for Lite 2 shown in Fig. 6 are slightly increased and correspondingly the LSR series for Lite 1 are slightly decreased, indicating the LSRs differ depending on the lite the external load is applied to. Most notably in Figs. 7 and 8, a significant difference in LSRs values exists between loading cases 1 and 4 , and 2 and 3 indicating the applied external load direction substantially affects the LSRs.

The LSR values shown in Figs. 5, 6, 7, and 8 for ASTM E1300 are 0.266 and 0.845 for Lite 1 (solid pink line) and Lite 2 (dashed pink line), respectively; 
Fig. 8 DIGU2 - iterative method load share ratios versus applied external uniform load magnitude for loading cases $5,6,7,8$

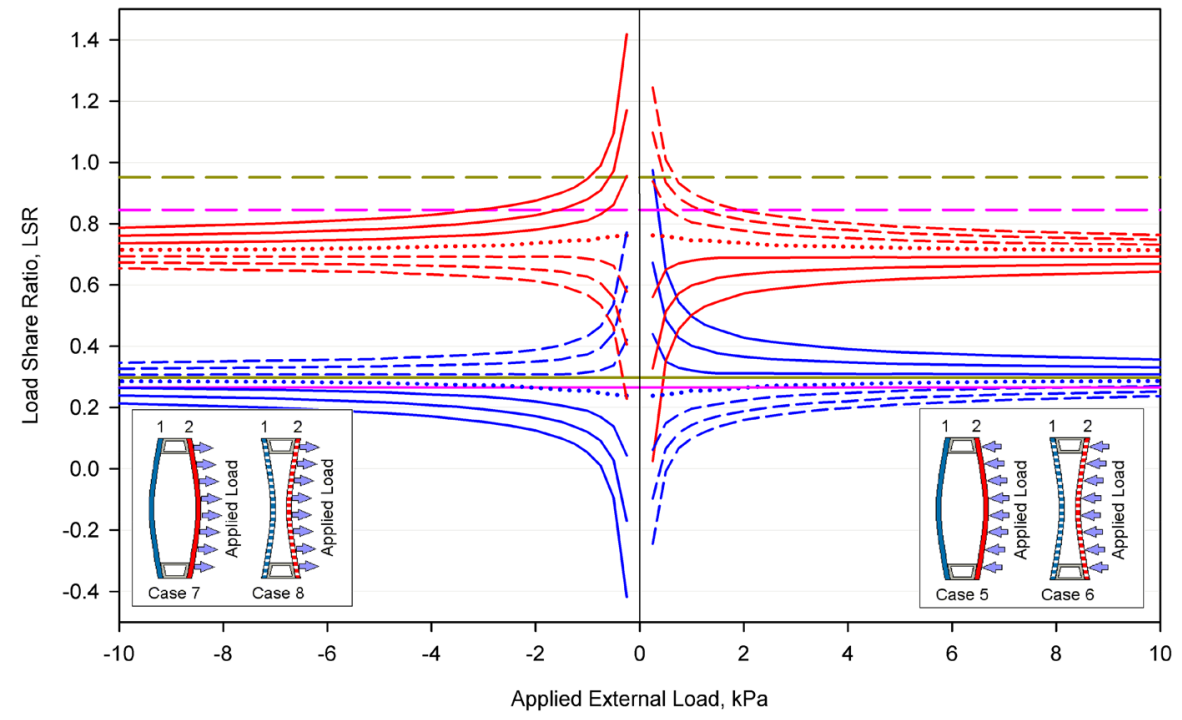

Fig. 9 DIGU3 - prEN 16612 method load share ratios versus applied external uniform load magnitude for loading cases $1,2,3,4$

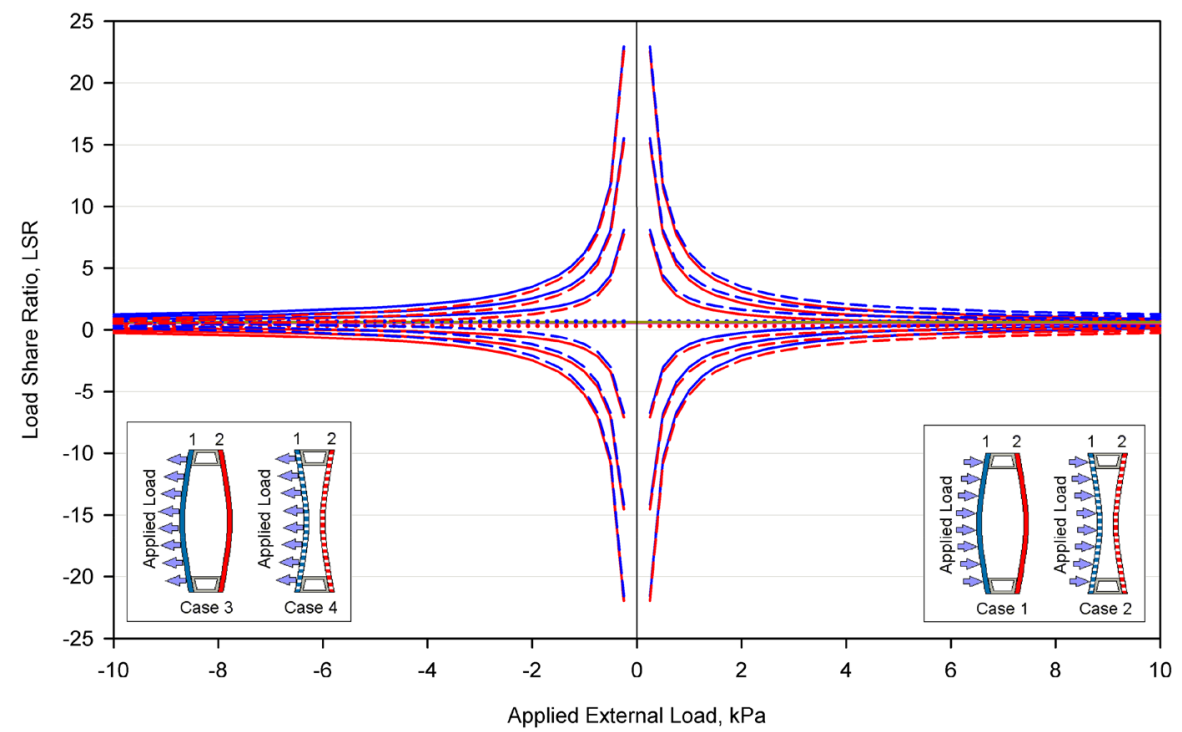

for AS 1288 the LSR values are 0.300 and 0.951 for Lite 1 (solid dark yellow line) and Lite 2 (dashed dark yellow line), respectively. Ideally, these values should be larger than those determined with the other methods, however they are only larger for Lite 2 when the applied external load is greater than $2.0 \mathrm{kPa}$. For Lite 1 , the iterative method LSR series shown in Figs. 7 and 8 are completely above both the ASTM E1300 and AS 1288 LSR series in loading cases 2, 3, 5 and 8 further supporting that the ASTM E1300 and AS 1288 methods do not sufficiently address many common loading conditions.

\subsection{IGU comparator: DIGU3}

DIGU3 represents a spandrel size symmetric DIGU comprised of thick lites for its rectangular dimensions to maintain optical consistency when positioned adjacent to a much larger unit requiring the larger lite thicknesses. Figure 9 shows the LSRs calculated using the prEN16612 method for loading cases 1, 2, 3, 4 . The trends of the LSR values are similar to those shown in Fig. 3 except the magnitudes are substantially larger, up to 23.0. The very large LSRs result from the increased air space pressure caused by the smaller vol- 
Fig. 10 TIGU1-prEN 16612 method load share ratios versus applied external uniform load magnitude for loading cases $1,2,3,4$

Fig. 11 TIGU1-iterative method load share ratios versus applied external uniform load magnitude for loading cases $1,2,3,4$
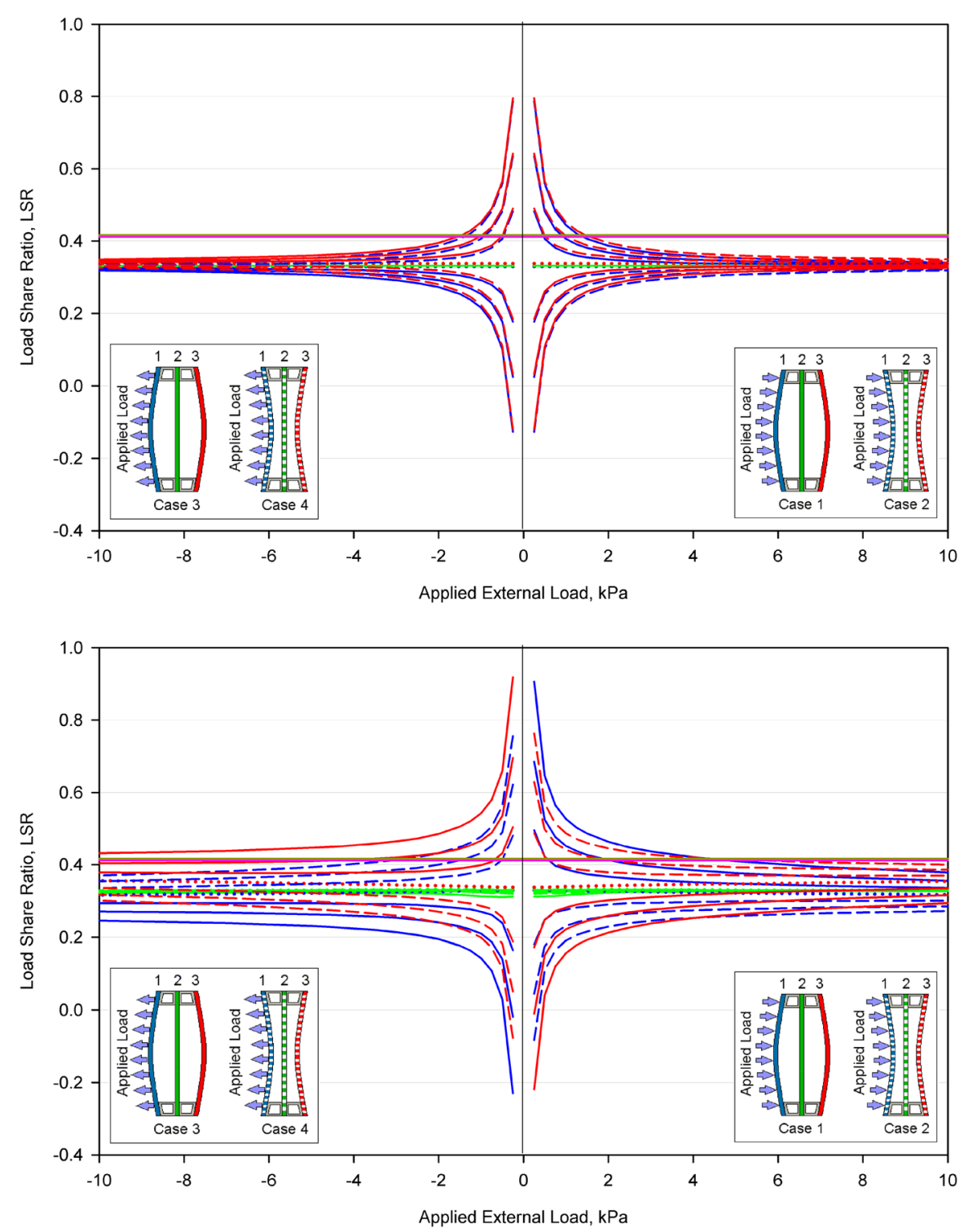

ume change due to the increases stiffness of the lites. DIGU3 provides insight into commonly used IGU constructions that appear more than adequate to resist the applied external load but may experience significantly increased loads due to environmental conditions causing the IGU unit to fail, either by permanent loads due to a change in elevation, high cyclic loadings due to temperature changes each day, or primary seal failures. The LSRs calculated using the iterative method are not presented as they do not significantly differ for the prEN16612 LSRs. Clearly the ASTM E1300 and AS 1288 methods do not adequately address this loading condition.

\section{Triple insulating glass unit comparators}

\subsection{IGU comparator: TIGU1}

TIGU1 represents a large commercial size symmetric TIGU. Figures 10 and 11 show the LSRs calculated using the prEN16612 and the iterative methods, respectively. Loading cases 5, 6, 7, 8 are not presented due to symmetry. An additional set of LSR series appears in these charts, shown in green, representing the middle lite. As with the symmetric DIGU comparators, the LSRs values in Fig. 10 between loading cases 1 and 2, and 3 and 4 are nearly equal and opposite with respect 
Fig. 12 TIGU2-prEN 16612 method load share ratios versus applied external uniform load magnitude for loading cases $1,2,3,4$
Fig. 13 TIGU2-prEN 16612 method load share ratios versus applied external uniform load magnitude for loading cases $5,6,7,8$
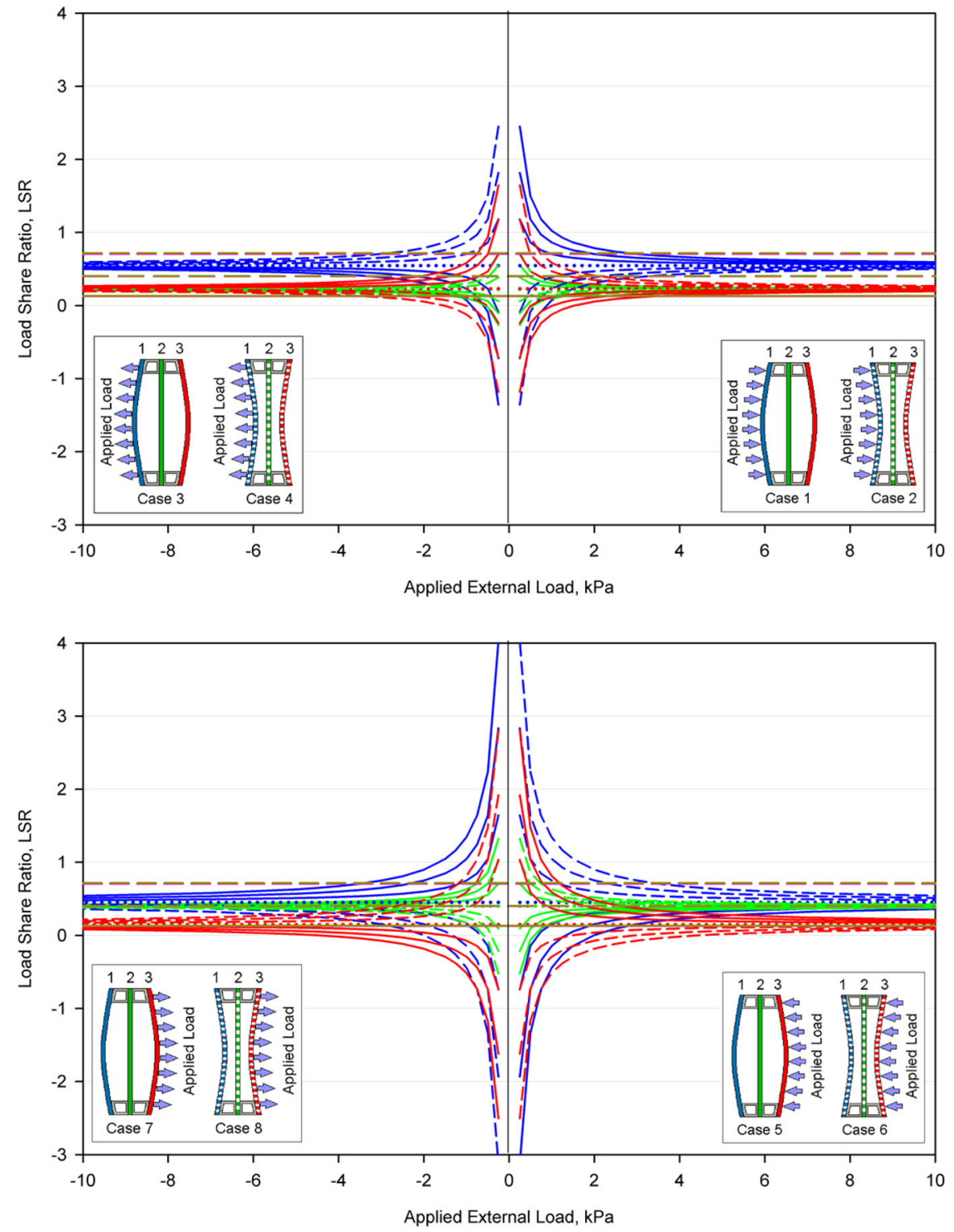

to the lites, indicating the sign of the $\mathrm{P}_{0}$ affects the LSR magnitudes, if only slightly for this comparator. As introduced with the DIGU comparators, loading cases 3 and 4 have exact mirror LSR values with respect to the applied external load magnitudes to loading cases 2 and 1, respectively. Indicating the same limitation also occurs in TIGUs. The LSRs values in Fig. 11 show similar trends to those presented in Fig. 10 and similar differences as shown between Figs. 3 and 4.

The LSR values calculated with ASTM E1300 and AS 1288 are 0.412 and 0.417 , respectively. Since the two methods are essentially equal, they are both represented with one set of pink lines as shown in Figs. 9 and 10. The ASTM E1300 and AS 1288 LSR values should be larger than those determined with the other methods, and they are for applied external load magnitudes greater than $2.0 \mathrm{kPa}$ in Fig. 9. However, as shown in Figs. 4, 10 also shows loading cases where ASTM E1300 and AS 1288 methods do not sufficiently encompass all possible variations in LSR values.

\subsection{IGU comparator: TIGU2}

TIGU2 represents a large commercial size asymmetric TIGU. Figures 12 and 13 present the eight loading cases 
Fig. 14 TIGU2-iterative method load share ratios versus applied external uniform load magnitude for loading cases 1, 2, 3, 4
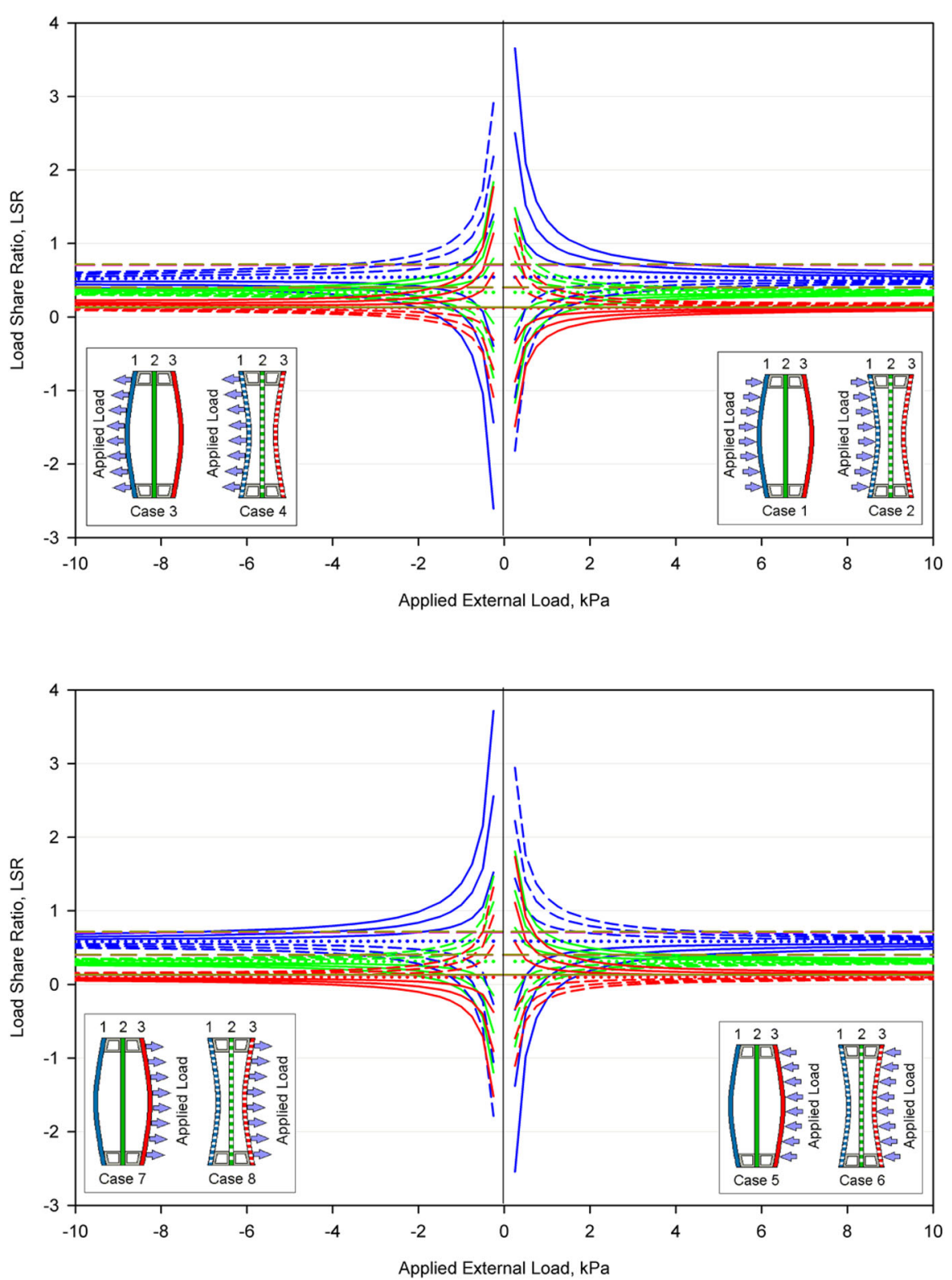

Fig. 15 TIGU2 - iterative method load share ratios versus applied external uniform load magnitude for loading cases $5,6,7,8$ for the prEN16612 LSRs and Figs. 14 and 15 present the eight loading cases for the iterative method LSRs. Surprisingly, the LSRs magnitudes do not increase proportionally with lite thicknesses as the thickness cubed method suggests. The prEN16612 LSRs shown in Fig. 12 for Lite 1 center around a LSR of 0.5 rather than 0.13 (solid pink line) as predicted by the ASTM E1300 and AS 1288 methods. Furthermore, the LSRs for Lite 2 and Lite 3 center on a LSR value of 0.22 indicating they carry a significantly smaller portion of the load than the 0.40 (short dash pink line) and 0.70 (long dash pink line) predicted by the ASTME1300 and AS 1288 methods, respectively. The prEN16612 LSRs shown in Fig. 13 display similar trends to those shown in Fig. 12, but the LRS for Lite 2 carries a larger portion of the load leaving Lite 3 with LSRs around 0.12. These trends are exactly counter to the proportions predicted by thickness cubed based methods, suggesting the thickness cubed based methods do not predict the behavior of asymmetric TIGUs well. The iterative method LSRs shown in Figs. 14 and 15 follow similar trends to those shown in Figs. 12 and 13, respectively. 
Fig. 16 TIGU3 - prEN 16612 method load share ratios versus applied external uniform load magnitude for loading cases $1,2,3,4$
Fig. 17 TIGU3 - prEN 16612 method load share ratios versus applied external uniform load magnitude for loading cases $5,6,7,8$
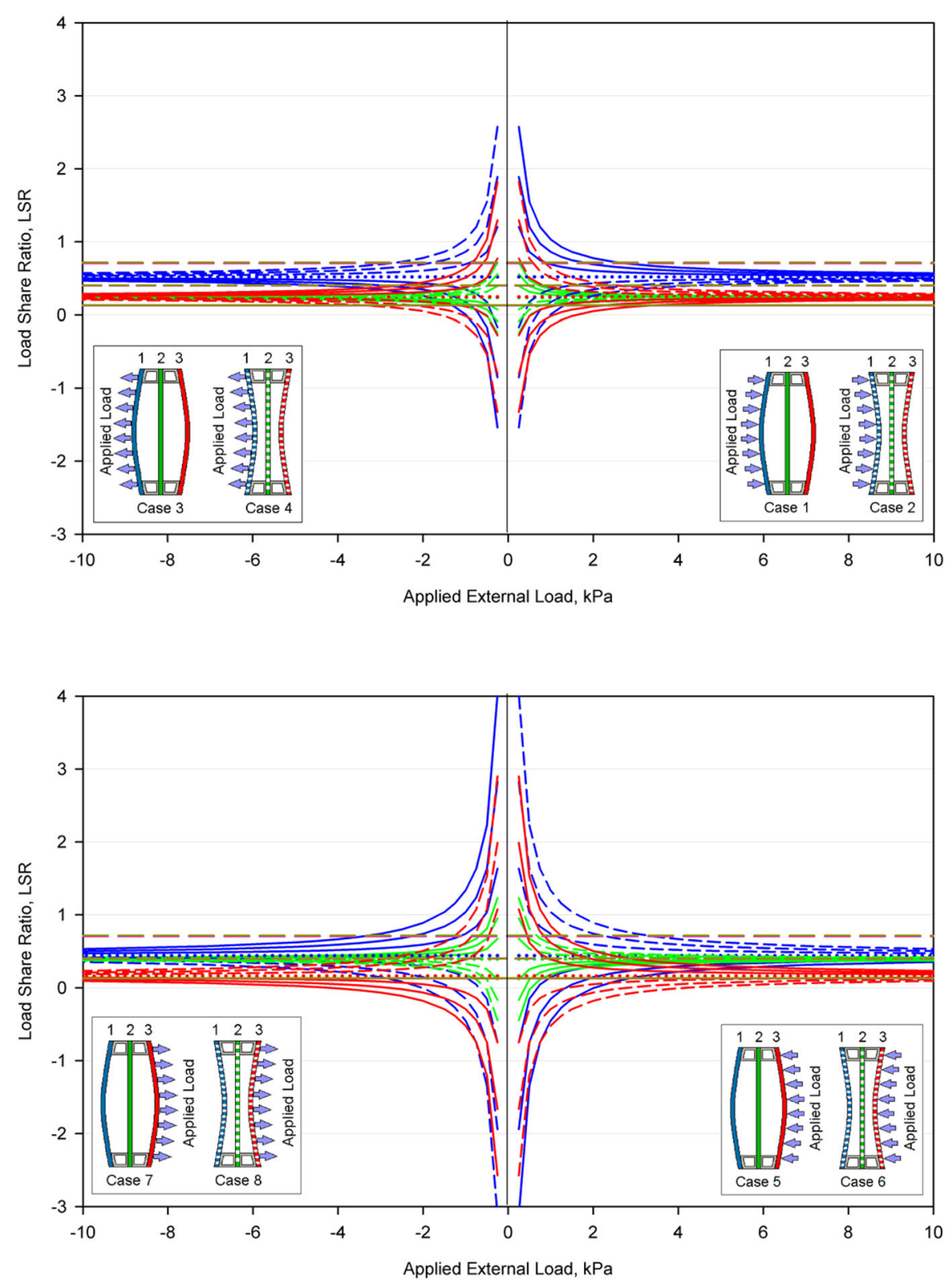

However, loading case 7 shows LSRs for Lite 1, Lite 2, and Lite 3 around 0.6, 0.3 and 0.1, respectively.

\subsection{IGU comparator: TIGU3}

TIGU3 represents a slight variation to TIGU2 due to switching the order of the lite thicknesses from smallest to largest to smallest, largest, and middle to explore if the order of the lite thicknesses affects the LSRs values. Figures 16 and 17 present the eight loading cases for the prEN16612 LSRs and Figs. 18 and 19 present the eight loading cases for the iterative method LSRs. Comparing the lites with the same thicknesses between the two orientations in TIGU2 and TIGU3, the prEN16612 LSRs shown in Figs. 16 and 17 have minor changes in magnitudes compared to those shown in Figs. 12 and 13 for TIGU2, indicating the prEN16612 method is not very sensitive to this change in lite thickness order. Comparing the iterative LSRs shown in Figs. 18 and 19, Lite 2 switches to the largest load carrying lite, which is consistent with the thickness cubed methods. 
Fig. 18 TIGU3 - iterative method load share ratios versus applied external uniform load magnitude for loading cases $1,2,3,4$

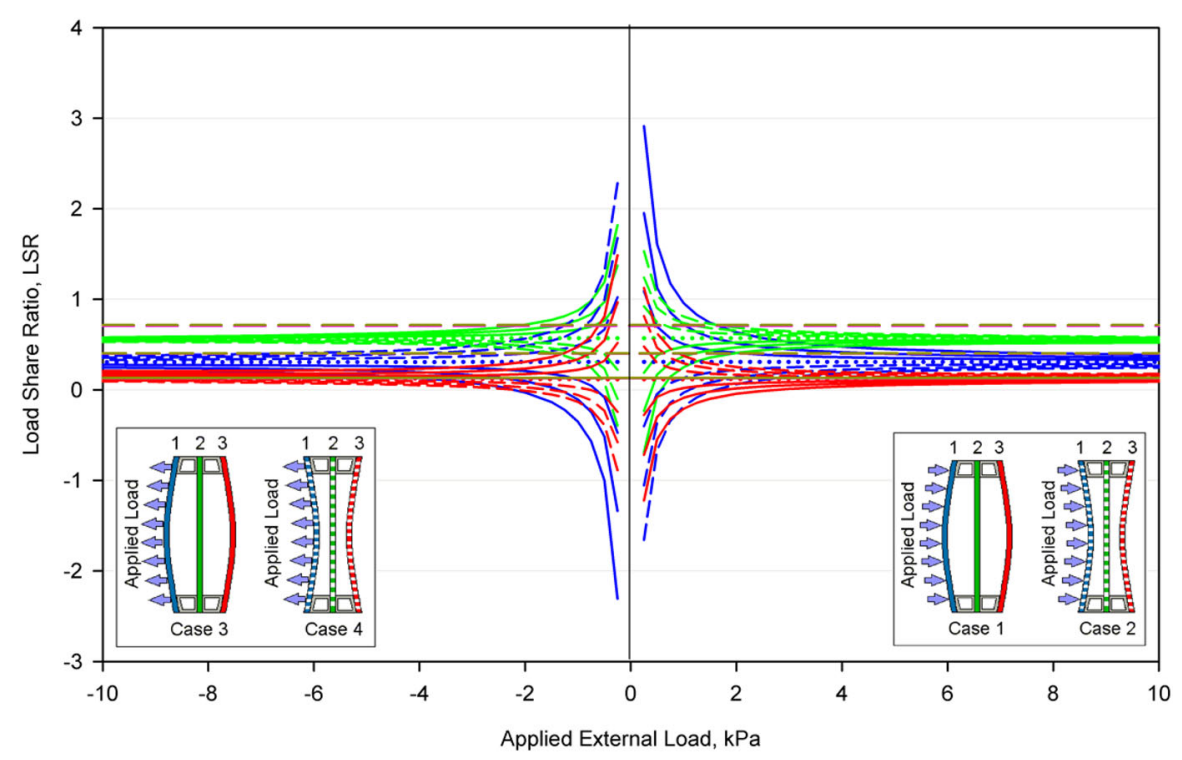

Fig. 19 TIGU3 - iterative method load share ratios versus applied external uniform load magnitude for loading cases $5,6,7,8$

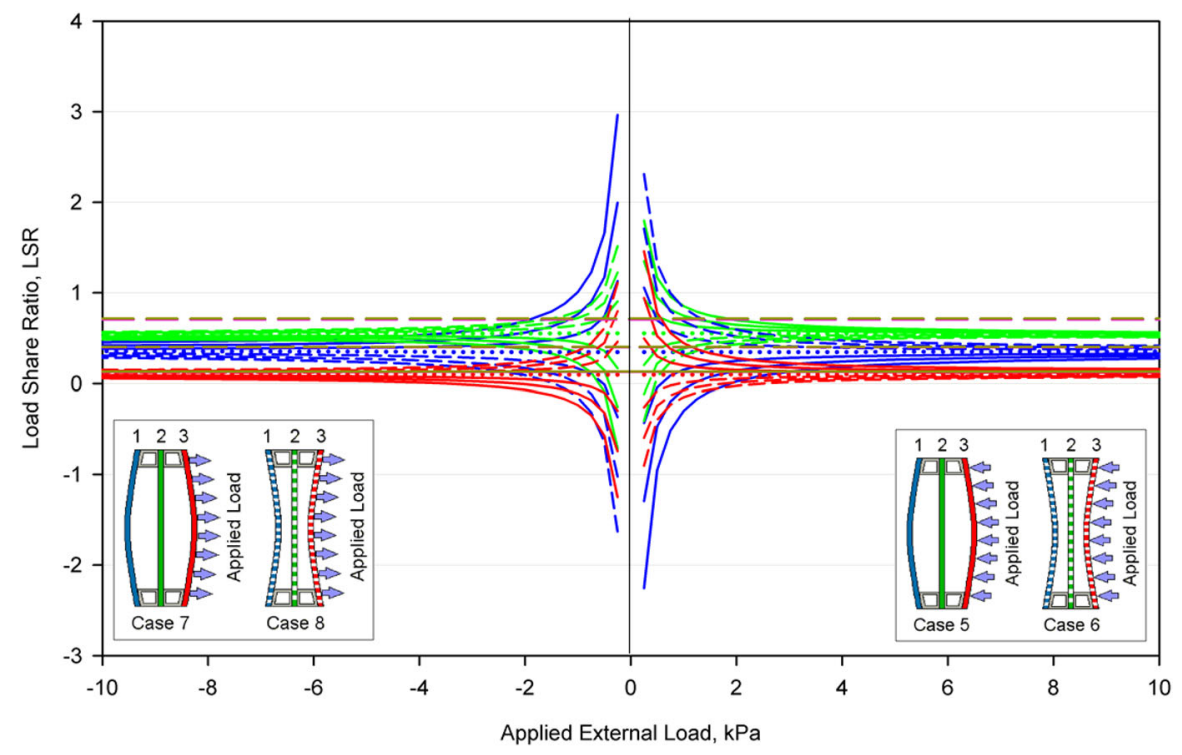

However, Lite 1 (the thinnest thickness) carries the next largest portion of the applied external load, leaving Lite 3 (the middle thickness) carrying the smallest portion of the applied external load, which is not consistent with the thickness cubed methods.

\section{Conclusions}

Even with the limited number of examples presented here, several trends have been elucidated. The most important finding is the load sharing of the lites comprising IGUs can be significantly affected by even small changes in $\mathrm{P}_{0}$ resulting from changes in the environmental conditions that create $\mathrm{P}_{0}$ across the IGU, more so for TIGUs. The thickness cubed methods including ASTM E1300 and AS 1288 do not adequately envelope many common IGU constructions exposed to $\mathrm{P}_{0}$. Furthermore, several examples advanced here show the thickness cubed methods are more than a $100 \%$ different, often with magnitudes smaller than predicted by the prEN 16612 and iterative methods. The prEN 
16612 does predict IGU load sharing more consistently with the iterative method but does not address all combinations of $\mathrm{P}_{0}$ sign and applied external load direction. Clearly, there are innumerable combinations of the listed factors that affect the load sharing of IGUs with environmental loads. Therefore, the presented examples should not be considered to encompass the extents of the possible variations but merely an indication that variations do exist. Additionally and likely a significant variable not addressed here is the edge conditions of the lite comprising the IGUs. The prEN 16612 and iterative methods are based on volume/load interactions for rectangular plates simply supported on four sides. However, the lites comprising IGU are generally not truly simply supported due to either: rotation resistance caused by the sealant between the spacer and the lites or the method of holding the IGU in the window frame. As these edge conditions will tend to cause the lites to behave in a stiffer manner than the simply supported case, the resulting lite volume changes will be different.

\section{Compliance with ethical standards}

Conflict of interest On behalf of all authors, the corresponding author states that there is no conflict of interest.

\section{References}

ASTM: Standard specification for flat glass. ASTM C 1036-11 ${ }^{\varepsilon 1}$, West Conshohocken, PA (2011)

ASTM: Standard practice for determining load resistance of glass in buildings. ASTM E 1300-12 $2^{\mathrm{a} \varepsilon 1}$, West Conshohocken, PA (2012)

Australian Standard (SAS): Glass in buildings-selection and installation, AS 1288.2-2006, Sydney, Australia (2006)

European Standard (EN): Glass in buildings-determination of the strength of glass panes by calculation and testing. prEN 16612 (2013)

Morse, S., Norville, H.: Comparison of non-linear to linear loadsharing of insulating glass units, GPD 2015. Tampere, Finland (2015)

Norville, H., Morse, S.: Load sharing and load resistance in tripleglazed insulating glass units, GPD 2011. Tampere, Finland (2011)

Siebert, G., Maniatis, I.: Tragende Bauteile aus Glas: Grundlagen, Konstruktion, Bemessung, Beispiele, Zweite Auflage. Wilhelm Ernst \& Sohn, Berlin (2012)

Vallabhan, C.V.G., Chou, G.D.: Interactive nonlinear analysis of insulating glass units. J. Struct. Eng. 112(6), 1313-1326 (1986) 